\title{
Huge Benign Teratoma of Middle Mediastinum - A Rare Case
}

\author{
Ravisagar Patel ${ }^{1}$, Abhinav Singh ${ }^{1}$, Roma Patel ${ }^{2}$, Archana Singh $^{2 *}$ \\ Sanjeev Devgarha ${ }^{3}$,Rajendra Mohan Mathur ${ }^{3}$ \\ ${ }^{I}$ (Resident M.Ch CTVS, Department of cardiothoracic and vascular surgery, SMS medical college, Jaipur India) \\ ${ }^{2}$ (Assistant Professor, Department of Anatomy, NIMS medical college, Jaipur, India) \\ ${ }^{2 *}$ (Clinical Associate Department of Obs.\&Gyn. Fortis Hospital, Jaipur ,India) \\ ${ }^{3}$ (Professor \& Head of Department of cardiothoracic and vascular surgery, SMS medical college, Jaipur India)
}

Abstract: A 28years old female presented with cough, dysponea and recurrent pulmonary infection. The CT and MRI Chest revealed middle mediastinal mass with pericardial attachment. She underwent midline sternotomy with successful enblock removal of the mass, the biopsy confirmed benign mature teratoma arising from pericardium. She was discharged on the $6^{\text {th }}$ post operative day, and is in regular follow up.

\section{Introduction}

Mediastinal teratomas are predominantly benign ${ }^{[1,2]}$. They are most common in young population with no sex predilection ${ }^{[2]}$. About $95 \%$ of the extragonadal germ cell tumors are located in the anterior mediastinum ${ }^{[2,3]}$. They represent approximately $15 \%$ of anterior mediastinal tumors in adults and $24 \%$ in children. Surgical resection where possible should be done for benign tumors as it provides better prognosis and survival rate.

\section{Case Report}

A 28 year multiparous, nonsmoker, female with no history of tuberculosis presented with productive cough, progressively worsening dyspnea, and recurrent pulmonary infections for last 6 months. Haematological investigation showed increased TLC. The chest X-ray showed opacity in the left hemithorax and collapse of the left lung. Pulmonary function tests were impaired: FVC 1.16 (37.2\% of predicted) and FEV1 1.04 (42.3\% of predicted). CT and MRI Scan revealed a mass of size $14 \times 10 \times 8 \mathrm{~cm}$ originating in the middle mediastinum and occupying almost whole of the left hemithorax compressing the heart, great vessels and hilar structure with mediastinal shift towards the right side and extending into anterior mediastinum. The mass showed heterogeneous density containing soft tissue elements, fat, cystic areas and foci of calcification. The fat planes between the mass and the pericardium was indistinct . Tumor markers ( $\alpha$-fetoprotein and $\beta$-human chorionic gonadotropin) were both raised. As the findings of CT scan was suggested of a benign tertoma, Surgical resection was planned. The patient underwent Midline Sternotomy and en-block removal of the mass along with left anterolateral pericardium. Postoperative period was unremarkable The biopsy showed a mass of $19 \mathrm{cmX} 15 \mathrm{cmX} 25 \mathrm{~cm}$ weighing $5 \mathrm{~kg}$. Multiple sections revealed cellular lesion consisting of admixture of ectodermal as well as mesodermal derived mature tissue including skin appendages, cartilage, marrow elements with no necrosis or glial tissue, findings consistence with benign mature mediastinal teratoma arising from the pericardium. She was discharged on $6^{\text {th }}$ postoperative day and is in our regular follow up.

\section{Discussion}

Germ cell tumors in the gonad are thought to originate from multipotential primitive germ cells. They are present usually in the gonads, rarely can occur in extragonadal locations, usually in or near the midline. Extragonadal germ cell tumors may arise from similar cells that are displaced along midline structures during their migration from the yolk endoderm to the gonad during early embryogenesis ${ }^{[4]}$.

The most common histologic type of mediastinal germ cell tumor is mature teratoma followed by seminoma ${ }^{[1]}$. They are composed of well-differentiated tissues derived from more than one of the three embryonic germ cell layers ${ }^{[4]}$.

Upto 53\% patients are asymptomatic tumor, discovered incidentally on chest radiographs obtained for other reasons ${ }^{[5]}$. Large tumors may produce symptoms due to compression of mediastinal structures. Patients may present with cough, dyspnea, chest pain, or pulmonary infection. Rarely, these tumors may rupture or erode into adjacent structures, such as the the pericardium, pleural space, the lung, or the tracheobronchial tree leading to pericardial effusions, pleural effusions, lipoid pneumonia, or expectoration of oily substances or hair (trichoptysis) may occur ${ }^{[4,6,7]}$. Tumor markers like Serum $\alpha$-fetoprotein and $\beta$-HCG levels must be measured if a teratoma or similar germ-cell tumor is suspected. An abnormal level of one or both markers is diagnostic ${ }^{[6]}$. 
Radiographically mature teratoma is rounded, sometimes lobulated mediastinal mass with the borders sharply marginated against the adjacent lung. Calcification (central, curvilinear, or peripheral) has been reported in approximately $20-43 \%$ of cases ${ }^{[5,7]}$. The radiographic visualization of teeth is pathognomonic of teratoma ${ }^{[8]}$. The presence of a fat-fluid level is considered specific for the diagnosis of teratoma but is seldom seen radiographically ${ }^{[9]}$. Computed tomography $(\mathrm{CT})$ is the modality of choice for the diagnostic evaluation of these tumors. It exquisitely shows the location, extent and intrinsic elements of the tumors including soft tissue, fat, fluid, and calcification. MR is sensitive in depicting the infiltration of the fat planes between the mass and the adjacent structures ${ }^{[10]}$. The treatment of mature teratoma consists of complete surgical excision of the mass. The prognosis is very good and 5-year survival rates approaches $100 \%$, in contrast to the prognosis of immature teratomas, which may exhibit an aggressive behaviour in adults and may have a poor prognosis ${ }^{[2]}$.

\section{Conclusion}

Benign Teratomas have a propensity for malignant transformation. Midline sternotomy provides a better access for masses attached to the percicardium, occupying anterior/middle mediastinum and should be the preferred approach. Surgical resection should be considered in young adults as it provides a better prognosis and survival rate for the patient.

\section{References}

[1]. C.R. Nichols Mediastinal germ cell tumors: clinical features and biologic correlates. Chest, 99 (1991), pp. 472-479

[2]. A.M. Marchevsky, M. Kaneko (Eds.), Surgical pathology of the mediastinum, C. Raven, New York (1984), pp. 117-159

[3]. J. Rosai, G.D. Levine Tumors of the thymus: germ cell tumors H.I. Firminger (Ed.), Atlas of tumor pathology: tumors of the thymus, fasc 13, ser 2, Armed Forces Institute of Pathology, Washington, DC (1976), pp. 182-190

[4]. F.Gonzalez-Crussi Extragonadal teratomas: hypotheses of origin W.H.Hartmann, W.R. Cowan (Eds.), Atlas of tumor pathology: extragonadal teratomas, fasc 18 ser 2, Armed Forces Institute of Pathology, Washington, DC (1982), pp.1-24

[5]. B.D. Lewis, I.W. Hurt, W.S. Payne, G.M. Farrow, R.H. Knapp, J.R. Muhm

[6]. Benign teratomas of the mediastinum J Thorac Cardiovasc Surg, 86 (1983), pp. 727-731

[7]. J.B. Kenny, H.M.L. Carty Infants presenting with respiratory distress due to anterior mediastinal teratomas: a report of three cases and a review of the literature

[8]. Br J Radiol, 61 (1988), pp. 241-244

[9]. U. Yeoman, H.R. Dalton, E.J. Adam Fat-fluid level in pleural effusion as a complication of a mediastinal dermoid, 1990 complication of a mediastinal dermoid: CT characteristics J Comput Assist Tomogr, 14 (1990), pp. 307-309

[10]. J. Dobranowski, L.F.W. Martin, W.E. Bennett CT evaluation of posterior mediastinal teratoma J Comput Assist Tomogr, 11 (1987), pp. 156-157

[11]. A.S. Fulcher, A.V. Proto, H. Jolles Cystic teratoma of the mediastinum: demonstration of fat/fluid level AJR, 154 (1990), pp. 259260

[12]. A. Drevelegas, P. Palladas, A. Scordalaki Eur Radiol, 11 (10) (2001), pp. 1925-1932

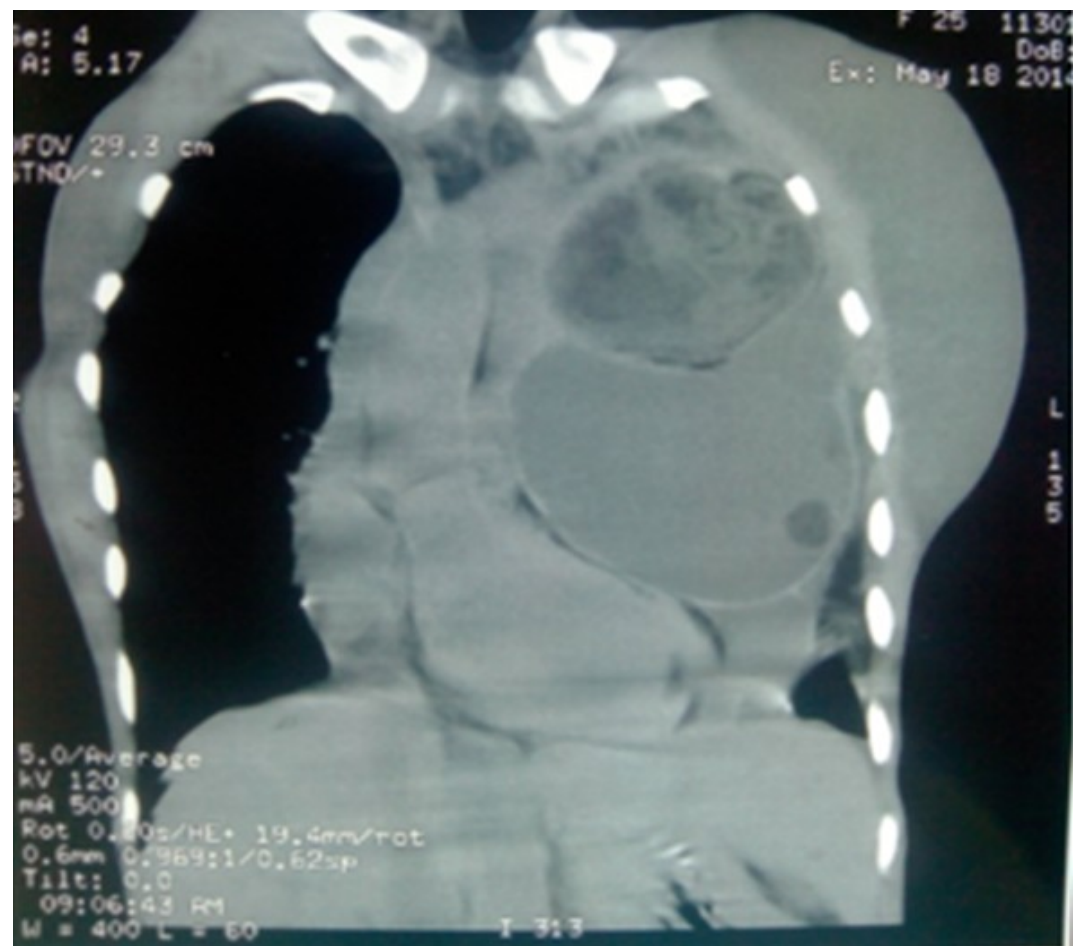

figure 1. CT chest showing large mediastinal mass with heterogeous shadows in it, and mass is compressing the middle mediastinal structures including heart 


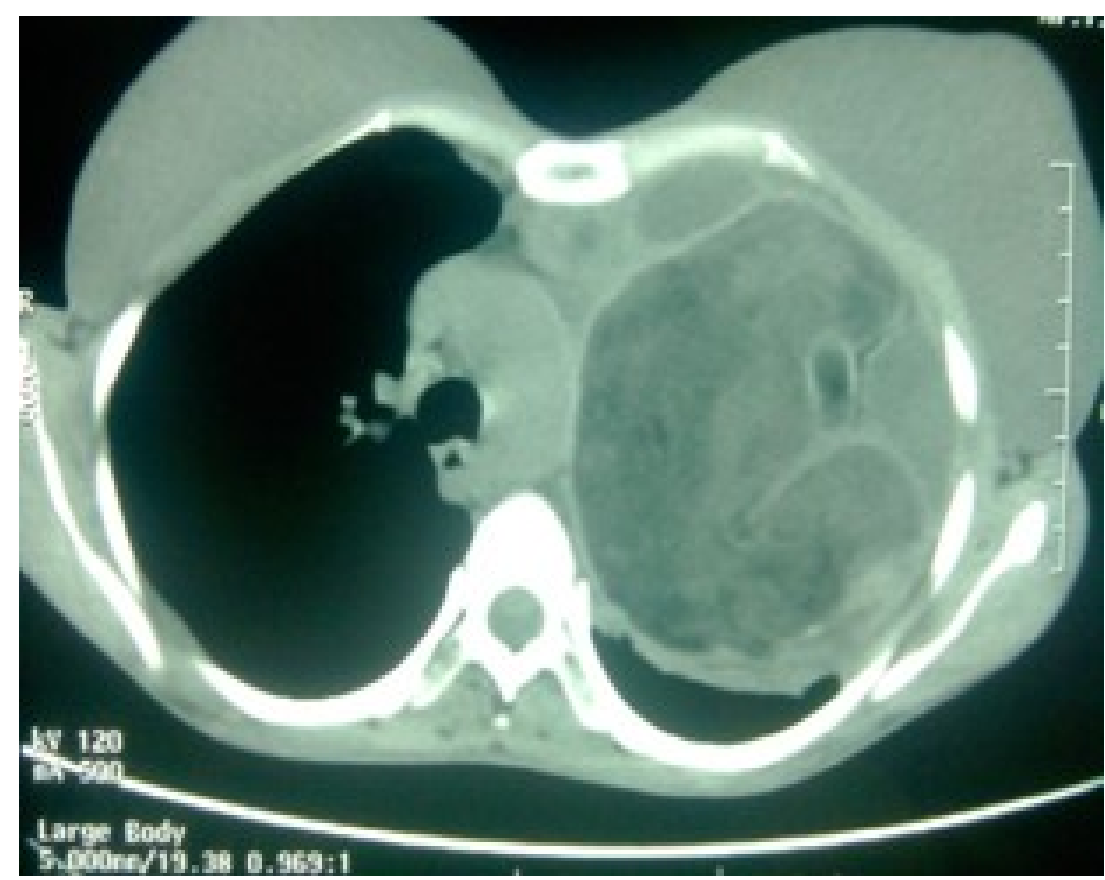

figure 2. Axial cut of CT chest showing the mass of heterogenous consistency and well defined margins, the medial margin blending with the pericardium

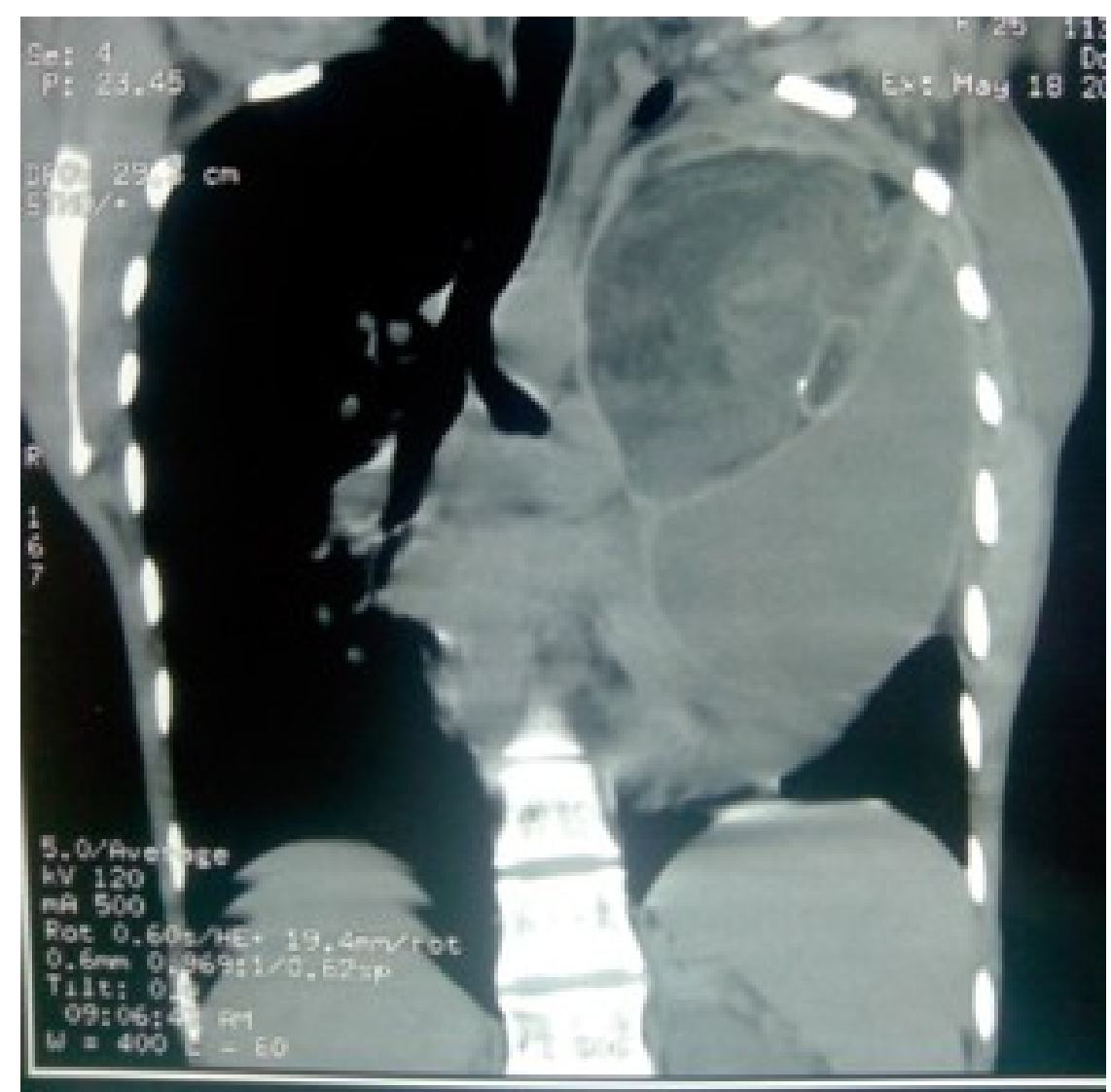

figure 3. CT chest showing the mass causing midline shift to right and compressing the trachea and pericardium 


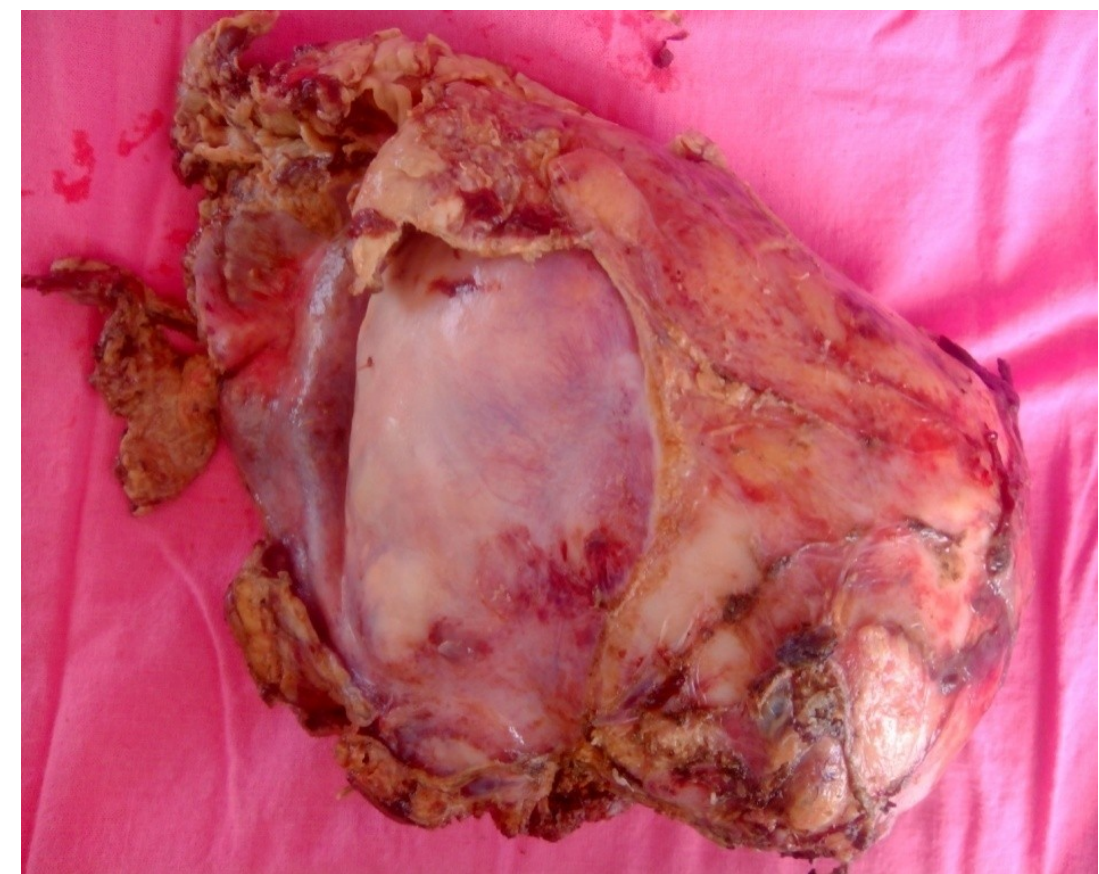

figure 4. Resected specimen cut opened to shoe the cavity of the mass and heterogenous nature of the mass.

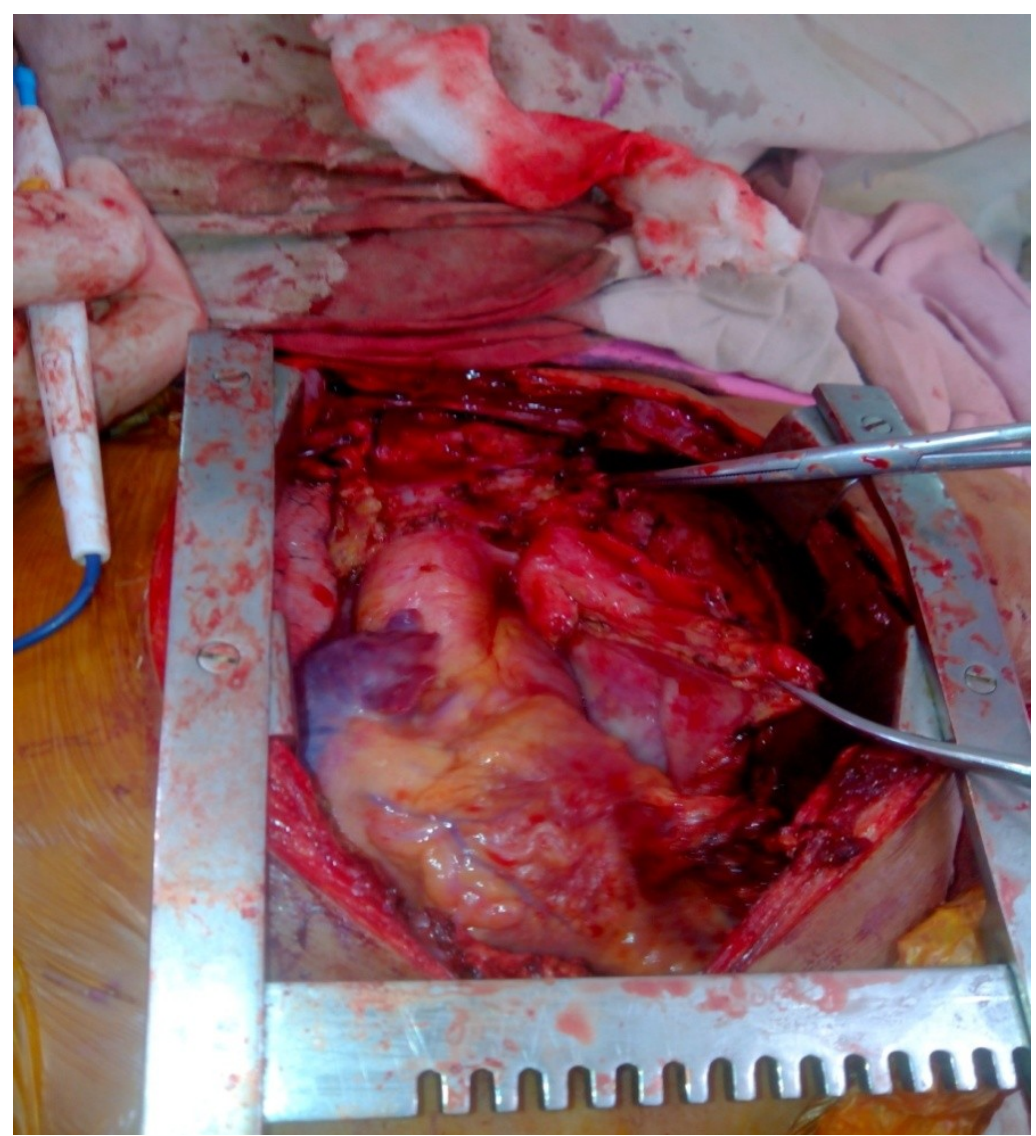

figure 5. Intraoperative photograph of midline sternotomy for the resection of the mediastinal mass. Mass shown abutting the pericardium from left side 


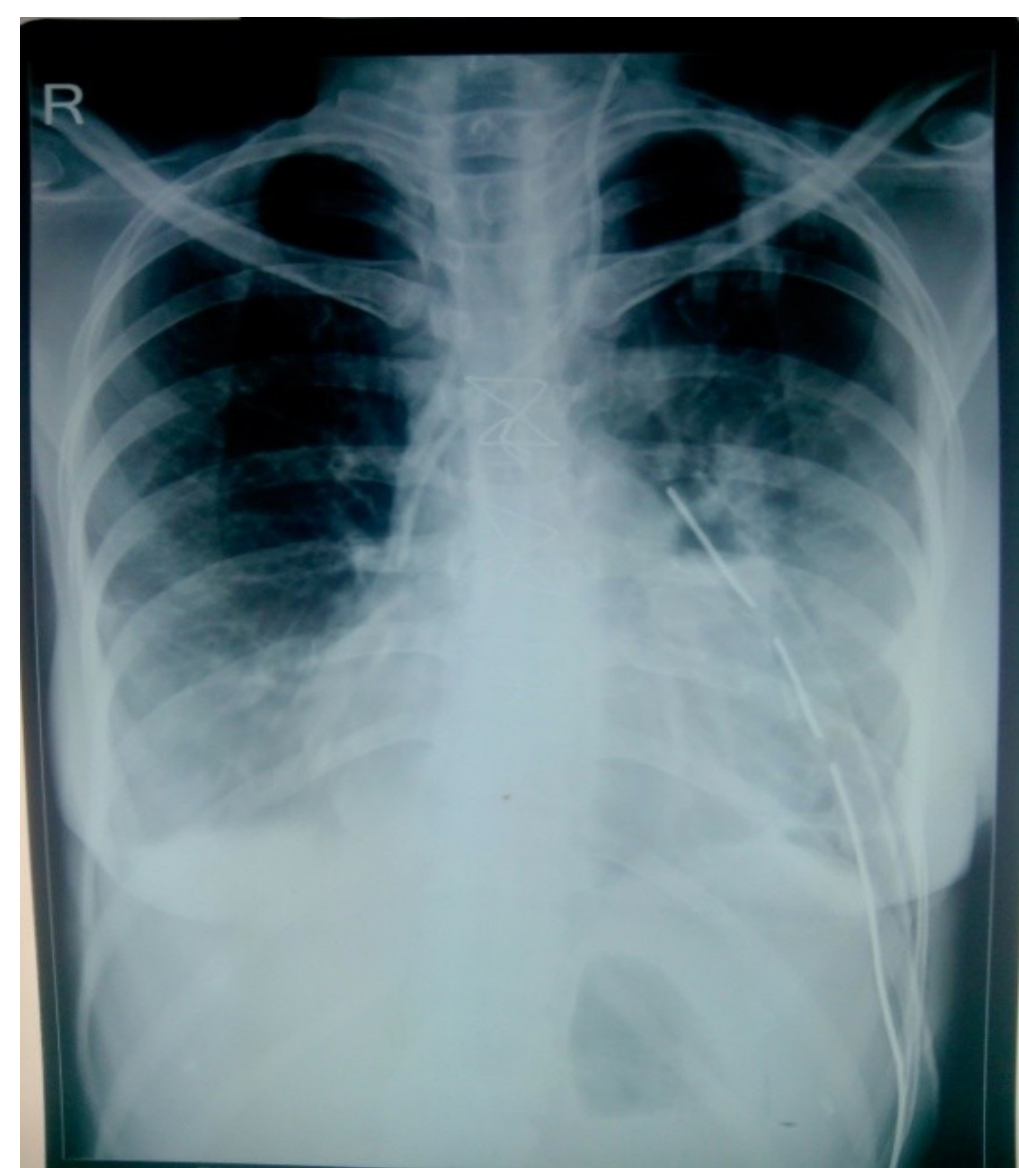

figure 6. Post-operative chest $\mathrm{x}$ ray showing the removal of entire mass and chest drain in situ . 